\title{
Handlungsfeld im Kommen
}

\author{
Im Zuge der Globalisierung nehmen die grenzüberschreitenden Transaktionen zu. \\ Damit treten die Norm- und Wertesysteme aber auch technische Standards mit- \\ einander in Wettbewerb. Zugleich steigt der Einfluss privater Akteure auf die \\ Standardsetzung. Anhand einer Typologie von Standards wird gezeigt, dass \\ neve Governance-Formen für eine nachhaltige Gestaltung dieser Dynamiken von \\ erheblicher Bedeutung sind.
}

I Von Ulrich Petschow und Britta Pielen nsbesondere im wirtschaftlichen Bereich ist festzustellen, dass nationale, vielfach hierarchische Formen der Regulierung an Bedeutung verlieren und andere Formen der Regulierung beispielsweise im Rahmen internationaler Organisationen an deren Stelle treten (können). Mit Bezug auf den Governancebegriff von Rosenau kann zwischen Government, das auf formeller, gesetzlich definierter und mit polizeilicher Gewalt ausgestatteter Autorität beruht, und Governance, welche als system of rule bei Abwesenheit von zentraler Durchsetzungsgewalt anzusehen ist, idealtypisch unterschieden werden (1). Im Kontext der Globalisierungsprozesse haben die Nationalstaaten zwar weiterhin eine wichtige Rolle, gleichwohl steht im Spannungsverhältnis von Fragmentierung und Integration die Bedeutung der Politik in Frage. Dies führt allerdings nicht dazu, dass ein durchgängiger Verlust an Autorität zu verzeichnen ist, vielmehr handelt es sich um eine Streuung bzw. Neuverteilung von Autorität. Brunson und Jacobson nehmen diese Beobachtungen zum Anlass, auf die zunehmende Bedeutung von Standards und Standardsetzungsprozessen insbesondere in der Wirtschaft zu verweisen (2). Standards sind in ihrer sehr allgemeinen Definition Verhaltensrichtlinien, die es ermöglichen das Verhalten einzelner Akteure bzw. Organisationen zu bewerten. Standards sind dabei explizit formuliert und mit Standardsetzern verbunden. Ihre Annahme ist zumeist freiwillig und die Standardsetzer haben vielfach keine, auf formaler Autorität beruhenden, Sanktionsmöglichkeiten. Dies bedeutet dann auch, dass der Wettbewerb und der Konflikt um Gestaltungssysteme zunimmt und auf dem Feld der Standards und Standardsetzungsprozesse ausgetragen wird.

Die Trennungslinien zwischen Normen (internalisierten Regeln), Direktiven (hierarchische Form

der Steuerung) und Standards sind zwar theoretisch gut beschreibbar, in der Praxis verwischen sich hingegen die Grenzen. So unterliegen den Standards mehr oder weniger explizit bestimmte Normen, sie explizieren diese Normen. Zugleich wird vielfach in Gesetzen und Verordnungen auf Standards Bezug genommen. Mithin sind damit auch private Organisationen und ihre Standardsetzungen und Bewertungen in staatliche Regulierungen eingebunden.

Einen systematisierten Überblick über den Beitrag, den Standards bei unterschiedlichen Externalitätenproblemen leisten können, entwickeln Abbott und Snidal. Tabelle 1 unterscheidet zwischen technologischen und regulatorischen Externalitäten einerseits und Netzwerk- und traditionellen Externalitäten andererseits.

\section{Vier relevante Externalitäten}

Zu I.: Die technologischen Interdependenzen stellen ein typisches Problem der Standardsetzung dar. Beispielsweise ist bei neuen Technologien die Standardisierung entscheidend für die Ausschöpfung von steigenden Skalenerträgen. Die Verbreitung dieser Technologien hängt letztlich davon ab, dass diese Standards anerkannt werden. Die Standardsetzer sind meist private Akteure. Machtverhältnisse spielen insofern eine Rolle, als mit der Annahme eines Standards Wettbewerbsvorteile verbunden sein können. Regierungen spielen vielfach eine wichtige Rolle indem sie diese Standards akzeptieren, inkorpo-

\begin{tabular}{|l|ll|}
\hline \multicolumn{3}{|c|}{ Tabelle I: Verschiedene Formen von Externalitäten } \\
\hline & Network Externalities & Traditional Externalities \\
\hline Technological Externalities & I. Technological Interconnectivity & III. Physical Externalities \\
\hline Regulatory Externalities & II. Transactional Interconnectivity & IV. Policy Externalities \\
\hline
\end{tabular}

Quelle: Abbott, K.W./ Snidal, D.: International "Standards" and International Governance. Working Paper Series 00.18, The Harris School 2000, S. 3.

rieren oder in internationalen Gremien unterstuitzen und diese Prozesse auch strategisch nutzen.

Zu II.: Ein zentrales Problem der Entwicklung der internationalen Märkte und der Ausschöpfung von Skalenerträgen sowie der Senkung der Transaktionskosten stellen unterschiedliche Standards in den einzelnen Nationalstaaten dar, die den Marktzutritt beschränken können und damit indirekt auch zu einer Bevorzugung der heimischen Unternehmen führen können. Dies stellte bei der Entwicklung des gemeinsamen Binnenmarktes der Europäischen Union ein Problem dar und ist auch weiterhin eines im Rahmen der Welthandelsorganisation (WTO). Bei den Akteuren handelt es sich einerseits um multinationale Unternehmen, die durch vereinheitlichte Regeln einen vereinfachten Marktzutritt erlangen können und andererseits auch um internationale Organisationen. Diese tragen im Sinne des freien Austausches zur Entwicklung eines level playing fields bei, indem in Verhandlungsprozessen mit den Nationalstaaten bestimmte Standards bzw. Guidelines entwickelt werden, die für die Nationalstaaten bindend werden. Diese Selbstbindung der Staaten führt vielfach zur so genannten negativen Integration, indem vorrangig hemmende Faktoren auf der Ebene von Mindeststandards beseitigt werden.

Zu III: Physische Externalitäten, also nichtkompensierte Schädigungen anderer Akteure, ergeben

h etwa durch das Verhalten einzelner Staaten bzw. der Unternehmen in diesen Staaten und verursachen gegebenenfalls Umweltprobleme in anderen Staaten oder tragen zu globalen Umweltproblemen bei. Durch die Entwicklung internationaler (Umwelt-) Verträge wird versucht diese Probleme zu überwinden. Dieser Ansatz der positiven Integration erweist sich in der Regel als deutlich schwieriger als der Abbau von Handelsschranken. Zentrale Akteure sind in diesem Falle meist Staaten und Nichtregierungsorganisationen. Es können aber auch Unternehmen sein, wie sich am Beispiel der FCKW Regulierung verdeutlichen lässt, bei deren Zustandekommen ein amerikanisches Chemieunternehmen eine wesentliche 
Rolle gespielt hat, da es über entsprechende Technologien verfügte und sich von daher einen Wettbewerbsvorteil versprechen konnte.

$\mathrm{Zu}$ IV: Politische Externalitäten ergeben sich, wenn sich die politisch festgesetzten Standards zwischen den Ländern unterscheiden und damit zu Marktzutrittshemmnissen werden. Im Unterschied zu den physischen Externalitäten werden durch die nationalen Standards im eigenen Land keine Umweltbelastungen in anderen Ländern verursacht. Die Differenz zu II. besteht vorrangig in der Begründung der Koordinierungsprobleme. So können diese Externalisierungen einerseits in deren strategischer Nutzung oder andererseits auf unterschiedlichen gesellschaftlichen Wertvorstellungen (Risikobewertung) beruhen. Beispielsweise kann der Konflikt um Hormonfleisch prinzipiell mit beiden Ansätzen begründet werden.

\section{Wichtige Rolle nichtstaatlicher Akteure}

Aus der angeführten Typisierung wird deutlich, dass die Setzung von Standards, wenn sie von internationalen Organisationen entwickelt werden, die Kooperation zwischen den Staaten erforderlich macht. Dabei kommen in der Regel solche Standards, die auf die Erweiterung der Märkte abzielen, leichter zu Stande als Standards, die zum Beispiel auf den Umweltschutz orientiert sind, mithin eine einschränkende Wirkung nach sich ziehen. Deutlich wird auch, dass in unterschiedlichen Standardsetzungsprozessen unterschiedlichste Akteure eine wesentliche Rolle spielen können. Dabei können Standards auch jenseits staatlicher Aktivitäten von privaten Akteuren entwickelt werden und damit einen erheblichen Einfluss gewinnen. Zweifelsohne hängt der Erfolg der Durchsetzung umweltorientierter Standards unter anderem von den jeweiligen Problemfeldern und konkreten politischen und marktlichen Kontexten ab. Der Wettbewerb der

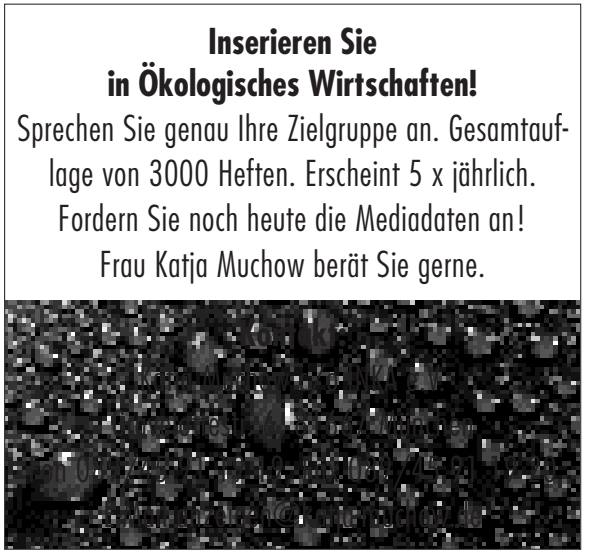

Standards ist vielfach intensiv, entscheidend ist dabei inwieweit die Festlegung von den Nutzern aufgegriffen werden.

Wie beschrieben erweisen sich Prozesse positiver Integration als deutlich schwieriger. In dieser Situation sind es gerade auch zivilgesellschaftliche Akteure bzw. Nichtregierungsorganisationen, die im Sinne einer civic regulation aktiv werden können. Ein Weg ist die Entwicklung von Standards, die letztlich nicht im Widerspruch zu Regelungen der WTO stehen, da diese Standards und die Übernahme der Standards auf freiwilliger Basis geschieht.

In der Folge werden drei Beispiele mit unterschiedlichen Akteuren als Treibern dargestellt, um einen konkreten Eindruck von den Dynamiken der Standards und Standardsetzungsprozesse zu erhalten.

\section{- Drei Praxisbeispiele}

Zivilgesellschaftliche Regulierungen: Das Forest Stewardship Council hat einen Standard für die nachhaltige Holznutzung und ein Zertifizierungssystem für entsprechend hergestellte Produkte entwickelt. Im Grundsatz verfügt diese Organisation über keinerlei formale Autorität, gleichwohl hat die Zertifizierung in Verbindung mit Marktentwicklungsmaßnahmen dazu beigetragen, dass sich ein Markt für entsprechende Produkte entwickelt hat. Das heißt diese Organisation hat über den Markt bzw. die Marktentwicklung für entsprechend zertifizierte Produkte überhaupt ihre Autorität gewinnen können. Diese Initiative hat dazu beigetragen, dass zugleich ein Wettbewerb um die nachhaltige Holznutzung entstanden ist, indem solche Unternehmen, denen die Regelungen des FSC zu strikt waren, eigene Standards entwickelt haben, die mehr oder weniger die bisherigen Praktiken legitimieren sollten (3).

Diffusion von staatlichen Standards: Ein bekanntes Beispiel stellen die kalifornischen Abgasstandards dar (california effect). Die besonderen Umweltprobleme der Region in Verbindung mit einer relativ hohen Umweltsensibilität führten zu der Entwicklung weitreichender Standards. Diese Standards wurden zum Vorbild für andere Regulatoren und wurden dementsprechend auch von anderen Ländern übernommen. Aufgrund der Bedeutung dieses Marktes für die Automobilindustrie waren die Unternehmen gezwungen, sich den dort vorgegebenen Regelungen anzupassen. Zugleich handelt es sich um ein mehr oder weniger ubiquitär vorhandenes Umweltproblem mit der Konsequenz, dass die kalifornischen Standards für die Automobilindustrie zu einer Orientierungsgröße wurden.

Internationale Organisationen: Im Rahmen der WTO werden grundsätzliche Regelungen des Waren- verkehrs festgelegt, in dem auf (zu interpretierende) allgemeine Regelungen abgehoben wird. Am Beispiel des Agreement on Sanitary and Phytosanitary Measures (SPS code) wird deutlich, wie stark damit zum einen die Festlegungen sind (und damit auch die Konflikte) und zum anderen die Standardentwicklungen anderer Organisationen einbezogen werden. So wird in diesem Abkommen ein enger Bezug zum Codex Alimentarius hergestellt, einer intergouvernementalen Organisation, die unter anderem durch die Welternährungs- und die Weltgesundheitsorganisation finanziert wird, um sowohl die Produzenten- als auch die Komsumenteninteressen im Hinblick auf sichere Ernährung zu vertreten. Konkret erweist sich diese Organisation in starkem Maße dominiert durch die Produzenteninteressen, die auch die Standardsetzungsprozesse dominieren. Die Staaten sind zwar vertreten, gleichwohl ist eine Dominanz der Unternehmen festzustellen. Die Mindeststandards, die in diesem Kontext entwickelt werden, werden im Rahmen der WTO als Messlatte für die Beurteilung von nationalen Standards herangezogen und führen damit zu einem direkten Einfluss auf die Möglichkeiten der nationalstaatlichen Regulierungen im Kontext der WTO.

Diese wenigen Beispiele machen die Dynamiken der Entwicklung deutlich. Diesen Dynamiken von Standardsetzungsprozessen sollte gerade im Sinne von global governance eine verstärkte Aufmerksamkeit zu Teil werden. Auf der einen Seite bestehen Chancen, durch private, zivilgesellschaftliche Ansätze der Standardentwicklung einen positiven Beitrag zur nachhaltigen Entwicklung zu leisten, und dies auch jenseits direkter staatlicher Einbindung. Auf der anderen Seite besteht ein erheblicher Bedarf, Standardisierungsprozesse etwa im Rahmen der WTO transparenter und demokratischer zu gestalten.

\section{Anmerkungen}

(1) Rosenau, J.N.: Towards an Ontology for Global Governance. In: Hewson, M. / Sinlaird, T.J. (eds.): Approaches to Global Governance Theory. New York 1999.

(2) Brunson, N./ Jacobson, B.: A World of Standards. Oxford 2000. (3) Vgl. hierzu auch Nill, J: Konkurrierende Standards für nachhaltige Forstwirtschaft. In: Ökologisches Wirtschaften Nr. 3/1999, S. 7

\section{Die Autorlnnen}

Ulrich Petschow ist Leiter des Forschungsfelds Umweltökonomie und -politik des Instituts für ökologische Wirtschaftsforschung (IÖW), Britta Pielen ist Wirtschaftswissenschaftlerin.

Kontakt: IÖW, Potsdamer Str. 105, 10785 Berlin. Tel. 030/884594-0, Fax 030/ 8825439,

E-Mail: Ulrich.Petschow@ioew.de 
(c) 20I0 Authors; licensee IÖW and oekom verlag. This is an article distributed under the terms of the Creative Commons Attribution Non-Commercial No Derivates License (http://creativecommons.org/licenses/by-nc-nd/3.o/), which permits unrestricted use, distribution, and reproduction in any medium, provided the original work is properly cited. 